\title{
Estabilidad de Antocianinas, Fenoles totales y Capacidad Antioxidante de Bebidas de Maíz Morado (Zea mays L.) y Uña de Gato (Uncaria tomentosa sp)
}

\author{
Edilberto Flores- Aguilar ${ }^{(1)^{*}}$ y Emille del P. Flores-Rivera ${ }^{(2)}$ \\ (1) Facultad de Ciencias e Ingenierías Biológicas y Químicas, Escuela Profesional de Ingeniería de Industria \\ Alimentaria, Univ. Católica de Santa Maria, Urb. San Jose s/n Umacollo, Arequipa - Perú. \\ (email: edicato@yahoo.es) \\ (2) Grupo de Investigación en Desarrollo de nuevos Alimentos Funcionales, Universidad Católica de \\ Santa (email:floresrivera_ep@hotmail.com)
}

${ }^{*}$ Autor a quien debe de ser dirigida la correspondencia

Recibido Ago. 17, 2017; Aceptado Oct. 16, 2017; Versión final Nov. 28, 2017, Publicado Abr. 2018

\begin{abstract}
Resumen
A partir de combinaciones de extractos de tusa de maíz morado y uña de gato, se elaboraron cuatro tipos de bebidas funcionales empleando stevia y sacarosa como edulcorantes. Se determinó sus características antioxidantes y estabilidad durante 86 días a 4 y $18{ }^{\circ} \mathrm{C}$ protegidas de la luz. La concentración de antocianinas se determinó con el método diferencial de $\mathrm{pH}$, fenoles totales por el método de Folin, la capacidad antioxidante por la reducción del ion cúprico y captura del radical catión del ácido 2,2'-azinobis- (3-etillbenzotiazolin-6-sulfonico). Las antocianinas se degradaron en menor grado en refrigeración que a temperatura ambiente siguiendo una cinética de primer orden. Los fenoles totales y capacidad antioxidante mostraron estabilidad con respecto al tiempo en el periodo evaluado independientemente de la temperatura de almacenamiento. Las bebidas presentaron resultados favorables en cuanto al contenido de antocianinas, y características sensoriales.
\end{abstract}

Palabras clave: capacidad antioxidante; maíz morado; uña de gato; stevia; antocianinas

\section{Stability of Anthocyanin Levels, Total Phenols and Antioxidant Activity of Beverages obtained from Purple Corn (Zea mays $L$.) and Cat's claw (Uncaria tomentosa sp)}

\begin{abstract}
:
Four types of functional beverages were prepared from purple corn cob and cat's claw extracts. For these formulations stevia and sucrose were used as sweeteners. The antioxidant properties and stability were analyzed for a period of 86 days at 4 and $18{ }^{\circ} \mathrm{C}$ protected from light. The concentration of anthocyanins, total phenols and antioxidant capacity were determined by the differential method of $\mathrm{pH}$, Folin Ciocalteu, Cupric reducing antioxidant capacity and capture of the cation radical 2,2'-azinobis-(3-ethylbenzothiazoline6 -sulfonic acid), respectively. Anthocyanin levels were degraded to a lower extent at $4^{\circ} \mathrm{C}$ compare to $18^{\circ} \mathrm{C}$ following a first order kinetics, total phenols and antioxidant activity showed stability for 86 days, and it was not affected by storage temperature. The final product showed favorable results regarding the amount of anthocyanins and sensorial characteristics.
\end{abstract}




\section{INTRODUCCIÓN}

La tendencia de los consumidores que ven importante el tema de salud, fomenta el desarrollo de nuevos tipos de bebidas empleando ingredientes naturales que contribuyan a prevenir la obesidad, diabetes entre otras dolencias. Diversos estudios señalan propiedades antioxidantes, antinflamatorias y anticancerígenas del maíz morado y uña de gato (Jing y Giusti, 2007; Amaral et al., 2009).Las antocianinas pertenecen al grupo de los flavonoides y son responsables de un amplio abanico de colores en vegetales, que van desde el azul, pasando por el púrpura, el violeta, el magenta y el rojo hasta el amarillo (Damodaran et al., 2010). El maíz morado es rico en antocianinas, su interés se ha incrementado debido a las propiedades de su color y potenciales beneficios a la salud, tales como regulación de la hipertensión arterial, prevención de la obesidad y diabetes, siendo una alternativa al uso de colorantes sintéticos (Giusti y Wrolstad, 2003).

Las antocianinas y otros polifenoles no solo están presentes en la cutícula del grano del maíz morado sino también en la tusa o coronta del maíz morado encontrándose en cantidades superiores. Sin embargo, las antocianinas se degradan por diversos factores tales como el incremento del pH, el aumento de temperatura y de la concentración de oxígeno. Las antocianinas son relativamente inestables, su mayor estabilidad ocurre bajo condiciones ácidas. La degradación de las antocianinas ocurre no solo durante la extracción del tejido vegetal sino también durante el procesado y conservación de los alimentos (Damodaran et al., 2010). La degradación de las antocianinas contenidas en la tusa del maíz morado durante en el proceso de extracción por acción de la temperatura sigue una reacción de primer orden (Yang et al., 2008). La eficiencia de extracción es influenciada por el comportamiento hidrofilico o lipofilico de estos compuestos y su afinidad al solvente empleado (Ramos et al., 2012). La estabilidad de las antocianinas en la elaboración de jugos resulta afectada por muchos factores tales como el tipo de antocianinas, de las materias primas y especialmente de la temperatura de almacenamiento (Hellstrom et al., 2013).

La uña de gato Uncaria tomentosa (Willd.) DC, es una planta medicinal con muchas aplicaciones terapéuticas. Utilizada por al menos 2000 años por muchas tribus de la Amazonia peruana, especialmente Ashaninka (Valerio y Gonzales, 2005; Pilarski et al., 2006). El extracto acuoso obtenido por cocción de la corteza de esta planta es usado en el tratamiento de enfermedades antiinflamatorias y anticancerígenas. Los extractos acuosos de Uncaria tomentosa son ricos en protoantocianidinas y flavanolignanos con efectos favorables en la salud en las enfermedades cardiovasculares y diabetes (Navarro et al., 2015). El uso de la stevia puede beneficiar a los diabéticos al reducir el consumo de azúcar, posee propiedades físico químicas favorables para su empleo en la industria alimentaria, muestra estabilidad térmica, tiene un poder edulcorante de 300 veces más que la sacarosa, es suficientemente estable en el rango de pH 3 a 9, entre otras propiedades (Rojas, 2009; Lemus et al., 2012). Su empleo viene extendiéndose cada vez más considerando la tendencia mundial en el consumo de productos dietéticos y naturales en remplazo del azúcar y los edulcorantes sintéticos (Rojas, 2009)

En la elaboración de bebidas funcionales resulta importante evaluar la estabilidad de las sustancias bioactivas presentes ya que el procesamiento suele afectar sus cualidades como tal y pueden disminuir en la vida útil del producto. El empleo de ingredientes naturales conteniendo sustancias bioactivas de interés propicia un aspecto particular en el procesamiento industrial y en el almacenamiento al tomar en cuenta la estabilidad de las mismas en las bebidas elaboradas. El uso de tusa de maíz morado, uña de gato y stevia como un edulcorante natural posibilitarían el desarrollo de nuevas bebidas funcionales con buenas características antioxidantes y sensoriales. El objetivo del estudio fue estudiar la estabilidad de antocianinas totales, fenoles totales y capacidad antioxidante en bebidas de Maíz Morado y Una de Gato durante el almacenamiento a dos temperaturas.

\section{METODOLOGIA}

La metodología contiene las siguientes subsecciones: una relacionada a las materias primas empleadas, otra referida al diseño de las formulaciones y elaboración de las bebidas, seguida por los análisis químicos, los modelos cinéticos empleados, la evaluación sensorial y el análisis estadístico.

\section{Materias primas}

Tusa de maíz morado variedad arequipeño se adquirió en un mercado local de la Provincia de Arequipa, Perú; la uña de gato provino del Departamento de Huánuco, Provincia de Pachitea, Distrito de Honoria, Fundo el cangrejal, Carretera campo Verde - Tournavista, Km 42, Perú; la stevia en polvo se compró en un mercado local en la ciudad de Arequipa.

\section{Diseño de las formulaciones y elaboración de las bebidas}

Se diseñó cuatro tipos de bebidas: a) Maíz morado - Azúcar, b) Maíz morado - Stevia, c) Maíz Morado - Uña de gato - Azúcar, d) Maíz morado - Uña de gato - Stevia. Las siglas para cada tipo de bebidas son: MMAZ, MMST, MMUGAZ, MMUGST, respectivamente. El extracto de uña de gato se preparó de la manera tradicional (Valerio y Gonzales, 2005)., se hirvió $20 \mathrm{~g}$ de tallo con la corteza (tronco parte aérea) de uña de gato en un 
litro de agua por 45 minutos, se decantó y se repuso el agua perdida en la evaporación Se preparó extractos acuosos de tusa de maíz haciendo hervir $40 \mathrm{~g}$ de tusa triturada por litro de agua (4\%) por $35 \mathrm{~min}$, con la finalidad de que al hacer la mezcla con los demás ingredientes, el contenido de maíz morado en las bebidas corresponda a un $3 \%$ de extracto de tusa en peso.

Para la elaboración de las bebidas que contienen uña de gato se mezcló el extracto de tusa de maíz morado con el extracto de uña de gato. La cantidad de extracto de uña de gato en la bebida fue de $20.3 \%$ volumen/volumen, esto es $60 \mathrm{~mL}$ de extracto preparado por envase de $296 \mathrm{~mL}$ de bebida. El volumen de 60 $\mathrm{mL}$ toma en cuenta su uso en la medicina tradicional (Valerio y Gonzales 2005). Se completó la diferencia con el extracto de tusa de maíz morado, se regulo el pH a 3.9 con ácido cítrico y se pasteurizo por 10 min a $92{ }^{\circ} \mathrm{C}$, se añadió en esta etapa las cantidades correspondientes de sacarosa para alcanzar $8^{\circ} \mathrm{Bx}$ y el equivalente de stevia de acuerdo al tipo de formulación diseñada. Se añadió 200 ppm de sorbato de potasio, se realizó el envasado en caliente en botellas GN-378 (JGO. 10OZ T/R) de $296 \mathrm{~mL}$, se enfrío de inmediato. Las bebidas obtenidas se almacenaron a $4^{\circ} \mathrm{C}$ y $18^{\circ} \mathrm{C}$, protegidas de la luz para su evaluación.

\section{Análisis químicos}

El contenido de antocianinas se determinó por el método diferencial de $\mathrm{pH}$ según el método propuesto por Wrolstad, 1976 y se expresó como mg de cianidina-3-glucosido/L, el contenido de Fenoles totales se determinó por el método de Folin y la Capacidad antioxidante por los métodos: CUPRAC (reducción del ion cúprico) , y ABTS (captura del radical catión del ácido 2,2'-azino-bis- (3-etillbenzotiazolin-6-sulfonico), según lo señalado por Guclu et al., 2006. Se empleó Trolox (ácido ( \pm )-6-hidroxi-2,5,7,8-tetrametil-croman-2carboxílico) como estándar en la elaboración de las curvas de calibración. La determinación de pH y acidez total titulable se realizó según el método de la AOAC 981.12/90 (AOAC, 1990), se utilizó un potenciómetro con precisión de 0.01 unidades de $\mathrm{pH}$. Los sólidos solubles totales (SST) se midieron en un refractómetro ABBE AR 12 Shmidt \& Haensen. Las medidas espectrofotométricas se realizaron en un espectrofotómetro Shimadzu UV-160 y las determinaciones de pH en un pH metro de mesa Jenway 3510.

Elaboradas las bebidas, la estabilidad se evaluó a dos temperaturas de almacenamiento 4 y $18^{\circ} \mathrm{C}$ y protegidas de la luz. El contenido de antocianinas, fenoles totales y capacidad antioxidante se determinó según los métodos señalados, para tal fin se tomaron al azar botellas conteniendo las bebidas elaboradas y mantenidas a 4 y $18{ }^{\circ} \mathrm{C}$, se realizaron además los análisis físico químicos y sensoriales durante el periodo de almacenamiento de 86 días para determinar su variación.

\section{Modelos cinéticos empleados}

Los modelos empleados fueron:

Modelo cinético de orden cero

$$
[A]=\left[A_{0}\right] \pm k t
$$

Modelo cinético de primer orden

$$
[A]=\left[A_{0}\right] e^{ \pm k t}
$$

Dónde: $\left[A_{0}\right]$ es la concentración inicial del atributo medido y $[A]$ la concentración del atributo medido a un tiempo $t$, y $k$ es una constante de velocidad.

Modelo de Arrhenius

$$
\begin{aligned}
& k=k_{0} \cdot \exp \left(-E_{a}\right) / R T \\
& L n k=L n k_{0}-\left(E_{a} / R\right)(1 / T)
\end{aligned}
$$

Dónde: $k$ es la constante de velocidad de la reacción, $k_{0}$ es el factor pre-exponencial, $E_{a}$ es la energía de activación, $R$ es la constante universal de los gases $(8,314 \mathrm{j} / \mathrm{Kmol})$ y $\mathrm{T}$ es la temperatura absoluta en $\mathrm{K}$.

Modelo $Q_{10}$

$$
Q_{10}=k_{T+10} / k_{T}
$$

Dónde: $T$ es la temperatura $\left({ }^{\circ} \mathrm{C}\right)$ y $k$ es la constante de velocidad de la reacción. 


\section{Evaluación sensorial}

Para evaluar el nivel de agrado se aplicó una escala hedónica (Anzaldua, 1994) cuyos puntos extremos y valoraciones fueron de 1 (Me disgusta mucho) a 7 (Me gusta mucho). La evaluación sensorial fue realizada con 8 jueces semientrenados (Espinoza, 2003). Se consideró una puntuación de 4.5 como límite favorable de satisfacción al producto. Para la vida útil se evaluó el sabor en una escala de 1 (No bebible) a 6 (Excelente), se consideró para tal fin una calificación de 3.5 como límite, debajo del cual se considera que el producto ha perdido sus características de calidad. La evaluación sensorial fue realizada por seis jueces entrenados (Espinoza, 2003).

\section{Análisis estadístico}

Las determinaciones de $\mathrm{pH}$ y acidez en las bebidas se realizaron por duplicado y las características antioxidantes por triplicado efectuándose 7 evaluaciones en el periodo de almacenamiento de 86 días. Se determinaron los coeficientes de correlación de los datos obtenidos. La evaluación sensorial se realizó en cada tipo de bebida elaborada, se efectuó un análisis de varianza y la prueba de Tuckey. Se utilizaron los programas Microsoft Excel y SPSS v 18.

\section{RESULTADOS Y DISCUSION}

En esta sección se presentan dos subsecciones, en la primera es relacionada a la estabilidad de antocianinas y en la segunda la estabilidad de los fenoles totales y capacidad antioxidante.

\section{Estabilidad de Antocianinas}

La Tabla 1 y Figura 1 muestra la variación del contenido de antocianinas y el porcentaje de retención (\% R) de antocianinas en las bebidas en el periodo estudiado, siendo la degradación mayor a temperatura ambiente $\left(18^{\circ} \mathrm{C}\right)$ que en refrigeración $\left(4^{\circ} \mathrm{C}\right)$. Los resultados experimentales del estudio cinético de la degradación de antocianinas presentes en las bebidas (Tabla 2), se ajustaron a una cinética de primer orden puesto que los coeficientes de correlación ( $r$ ) entre el logaritmo de la concentración de antocianinas y el tiempo de almacenamiento tuvieron valores cercanos a uno mucho más que los coeficientes de correlación hallados para una cinética de orden cero a las temperaturas estudiadas.

Tabla 1: Contenido de Antocianinas de las bebidas elaboradas en el almacenamiento

\begin{tabular}{|c|c|c|c|c|c|c|c|c|}
\hline \multirow{2}{*}{$\begin{array}{c}\text { Tiempo } \\
\text { (d) }\end{array}$} & \multicolumn{2}{|c|}{$M M A Z$} & \multicolumn{2}{|c|}{$M M S T$} & \multicolumn{2}{|c|}{ MMUGAZ } & \multicolumn{2}{|c|}{ MMUGST } \\
\hline & $m g / L$ & $(\% R)$ & $m g / L$ & $(\% R)$ & $m g / L$ & $(\% R)$ & $m g / L$ & $(\% R)$ \\
\hline \multicolumn{9}{|c|}{$4^{\circ} \mathrm{C}$} \\
\hline 3 & $150.4 \pm 1.18$ & 100.0 & $137.8 \pm 1.16$ & 100 & $144.0 \pm 1.14$ & 100.0 & $135.7 \pm 1.14$ & 100 \\
\hline 17 & $131.5 \pm 1.10$ & 87.4 & $134.5 \pm 1.15$ & 97.6 & $140.3 \pm 1.16$ & 97.4 & $128.6 \pm 1.17$ & 94.8 \\
\hline 29 & $129.4 \pm 1.19$ & 86.0 & $129.4 \pm 1.17$ & 93.9 & $133.5 \pm 1.17$ & 92.7 & $127.3 \pm 1.17$ & 93.8 \\
\hline 43 & $129.0 \pm 1.17$ & 85.8 & $128.6 \pm 1.18$ & 93.3 & $133.1 \pm 1.15$ & 92.4 & $126.1 \pm 1.18$ & 92.9 \\
\hline 57 & $124.9 \pm 1.18$ & 83.0 & $123.6 \pm 1.15$ & 89.7 & $131.9 \pm 1.16$ & 91.6 & $122.3 \pm 1.16$ & 90.1 \\
\hline 73 & $122.7 \pm 1.16$ & 81.6 & $121.9 \pm 1.18$ & 88.5 & $131.1 \pm 1.17$ & 91.0 & $120.2 \pm 1.19$ & 88.6 \\
\hline 86 & $118.1 \pm 1.14$ & 78.5 & $113.2 \pm 1.14$ & 82.1 & $124.0 \pm 1.18$ & 86.1 & $114.0 \pm 1.18$ & 84.0 \\
\hline \multicolumn{9}{|c|}{$18^{\circ} \mathrm{C}$} \\
\hline 3 & $150.4 \pm 1.15$ & 100 & $137.8 \pm 1.16$ & 100 & $144.0 \pm 1.13$ & 100 & $135.7 \pm 1.17$ & 100 \\
\hline 17 & $126.5 \pm 1.16$ & 84.1 & $129.8 \pm 1.15$ & 94.2 & $130.2 \pm 1.14$ & 90.4 & $127.3 \pm 1.15$ & 93.8 \\
\hline 29 & $124.9 \pm 1.14$ & 83.0 & $121.5 \pm 1.18$ & 88.2 & $122.7 \pm 1.12$ & 85.2 & $119.8 \pm 1.16$ & 88.3 \\
\hline 43 & $114.4 \pm 1.19$ & 76.0 & $114.8 \pm 1.17$ & 83.3 & $114.8 \pm 1.15$ & 79.7 & $119.4 \pm 1.17$ & 88.0 \\
\hline 57 & $111.0 \pm 1.18$ & 73.8 & $107.3 \pm 1.16$ & 77.9 & $114.8 \pm 1.16$ & 79.7 & $106.5 \pm 1.14$ & 78.5 \\
\hline 73 & $102.7 \pm 1.14$ & 68.3 & $108.5 \pm 1.18$ & 78.7 & $111.0 \pm 1.15$ & 77.1 & $107.7 \pm 1.17$ & 79.4 \\
\hline 86 & $98.9 \pm 1.15$ & 65.8 & $98.5 \pm 1.16$ & 71.5 & $100.9 \pm 1.14$ & 70.1 & $95.6 \pm 1.16$ & 70.4 \\
\hline
\end{tabular}



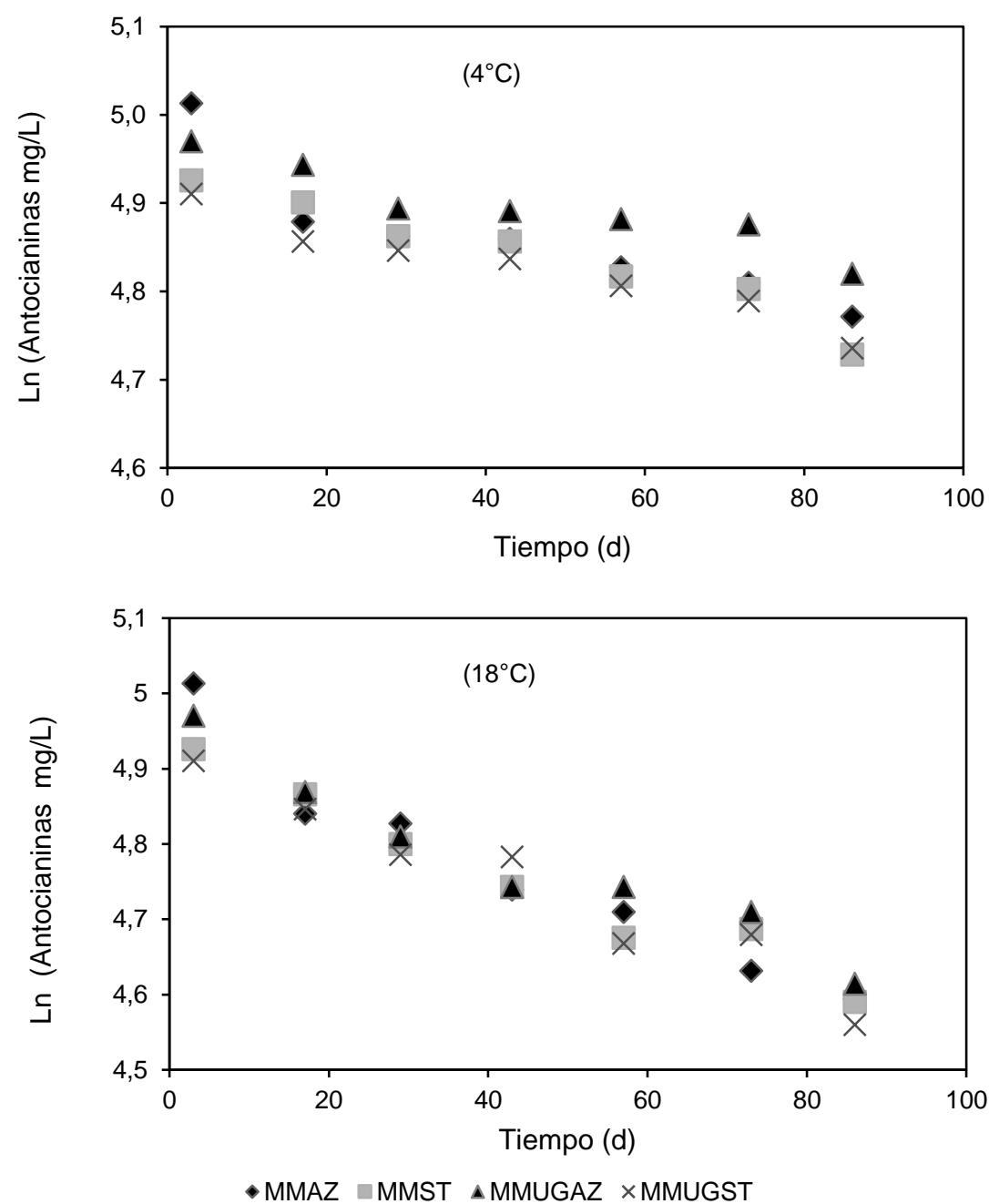

Fig.1: Variación del contenido de antocianinas en almacenamiento a 4 y $18^{\circ} \mathrm{C}$

Tabla 2: Parámetros cinéticos de degradación de Antocianinas

\begin{tabular}{|l|c|c|c|c|c|c|}
\hline \multirow{2}{*}{ Bebida } & \multicolumn{5}{|c|}{ Parámetros } \\
\cline { 2 - 7 } & \multicolumn{3}{|c|}{$4^{\circ} \mathrm{C}$} & \multicolumn{1}{c|}{$18^{\circ} \mathrm{C}$} \\
\cline { 2 - 7 } & $k\left(d^{-1}\right)$ & $t_{1 / 2}(d)$ & $r$ & $k\left(d^{-1}\right)$ & $t_{1 / 2}(d)$ & $r$ \\
\hline MMAZ & $0.0023 \pm 0.001$ & 301 & 0.9 & $0.0046 \pm 0.002$ & 150 & 0.97 \\
\hline MMST & $0.0021 \pm 0.002$ & 330 & 0.97 & $0.0038 \pm 0.001$ & 182 & 0.98 \\
\hline MMUGAZ & $0.0015 \pm 0.000$ & 462 & 0.94 & $0.0037 \pm 0.000$ & 187 & 0.97 \\
\hline MMUGST & $0.0018 \pm 0.001$ & 385 & 0.97 & $0.0039 \pm 0.001$ & 178 & 0.97 \\
\hline
\end{tabular}

El estudio de los parámetros cinéticos de degradación de antocianinas (Tabla 2) en las bebidas señala para el caso de las antocianinas valores de las constantes de velocidad de degradación $(k)$ mayores en las bebidas almacenadas a temperatura de $18^{\circ} \mathrm{C}$ que a $4^{\circ} \mathrm{C}$. Las energías de activación (Ea) calculadas fueron: de 32.0, 27.4, 41.7 y $35.7 \mathrm{KJ} / \mathrm{mol}$ para las bebidas MMAZ, MMST, MMUGAZ y MMUGST respectivamente. Para que ocurra una reacción una molécula debe poseer una cantidad mínima de energía, llamada energía de activación (Chang, 2008), lo cual implica que las bebidas de MMAZ y MMST son más sensibles a degradarse al tener menores valores de energía de activación. En estudios de degradación del color ASTA en pulpa de ají escabeche López et al., 2009 determinaron un valor de energía de activación de $51.94 \mathrm{KJ} / \mathrm{mol}$ mayor al de la degradación de pulpa de ají verde $(25.02 \mathrm{KJ} / \mathrm{mol})$ y ají rojo $(24.80 \mathrm{KJ} / \mathrm{mol})$ determinados por otros investigadores, efectuando las comparaciones López et al., 2009, concluyeron que la degradación del color ASTA en la pulpa de ají escabeche presentaba una menor sensibilidad al efecto de la temperatura de calentamiento. 
Los valores de $\mathrm{Q}_{10}\left(8-18^{\circ} \mathrm{C}\right)$ determinados fueron de $1.65,1.53,1.91$ y 1.74 para las bebidas MMAZ, MMST, MMUGAZ, MMUGST. Los valores de $Q_{10}$ representan la sensitividad de la reacción a un cambio de temperatura de $10^{\circ} \mathrm{C}$, lo cual implica que las bebidas de MMAZ y MMST son más sensibles al incremento e temperatura en $10^{\circ} \mathrm{C}$. Los resultados de $k$, Ea, Q 10 guardan relación con respecto a la estabilidad de las antocianinas en las bebidas. La bebida de MMUGAZ presenta un menor valor de $k$, mayor valor de Ea, Q $10 \mathrm{y}$ mayor tiempo de vida media $\left(\mathrm{t}_{1 / 2}\right)$, siendo la bebida que muestra una mayor estabilidad que las demás.

Diversos estudios realizados en otras matrices alimentarias muestran resultados similares, así Martínez et al., 2011, encontraron en jugos y concentrados de agraz, evaluados durante 4 semanas a 4,17 y $37^{\circ} \mathrm{C}$ una cinética de estabilidad térmica para las antocianinas de primer orden, siendo los parámetros cinéticos favorables a las condiciones de refrigeración. El tiempo de vida media fue más corto para las antocianinas en jugos de bayas a temperatura ambiente que en refrigeración (Hellstrom et al., 2013). Arrazola et al., 2014 determinaron, que la estabilidad de las antocianinas extraídas de berenjena previamente deshidratadas y aplicadas en la elaboración de bebidas fue mayor a $4^{\circ} \mathrm{C}$ que a $25^{\circ} \mathrm{C}$ durante 40 días de almacenamiento. De igual forma Hurtado y Pérez, 2014, en el estudio de estabilidad de extractos obtenidos a partir de la cascara de capulí, las cinéticas de degradación para las antocianinas en un tiempo de 90 días de evaluación fueron de primer orden. Además muchos estudios han determinado la inestabilidad de las antocianinas frente a la temperatura, pH, luz (Laleh et al., 2006). Adicionalmente se ha establecido que algunas especies contienen antocianinas más resistentes; así antocianinas aciladas contenidas en algunas especies como boniatos rojos son más resistentes que el maíz morado y en uvas rojas con mayor contenido de antocianinas no aciladas (Cevallos y Cisneros, 2004; Malien et al., 2001).

\section{Estabilidad de Fenoles totales y Capacidad antioxidante}

Las Figuras 2, 3 y 4 muestran los resultados del contenido de fenoles totales y de la capacidad antioxidante (CA) en el periodo evaluado de almacenamiento. Los valores de $k$ determinados fueron bajos y los coeficientes de correlación estuvieron comprendidos entre 0.14 y 0.94 . Siendo pequeños los valores de $k$ evidencian, la poca variabilidad de la concentración de fenoles totales y capacidad antioxidante en el tiempo según se aprecia en las figuras en mención.
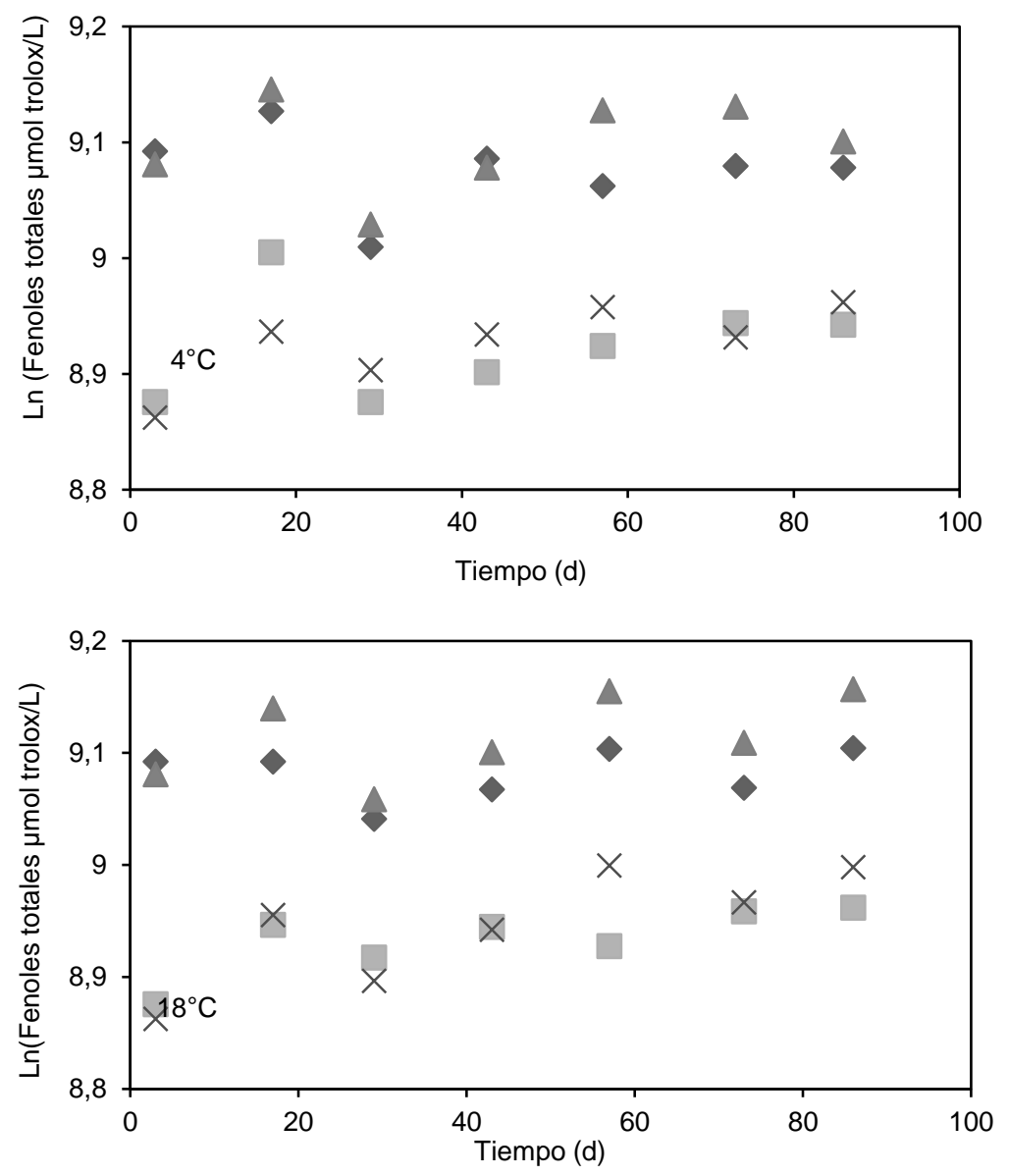

- MMAZ $\square$ MMST $\triangle$ MMUGAZ × MMUGST

Fig. 2: Estabilidad de los Fenoles totales en almacenamiento a 4 y $18^{\circ} \mathrm{C}$ 
Los resultados encontrados difieren al de otros estudios tal es el caso de la estabilidad de bebidas de borojó las cuales fueron evaluadas a 4,17 y $37^{\circ} \mathrm{C}$ durante 17 días y que muestran cinéticas de primer orden en la degradación de los compuestos fenólicos y de capacidad antioxidante, siendo la degradación mayor cuando la temperatura se incrementa (Camelo y Sotelo, 2012). Sin embargo debe mencionarse la ausencia de antocianinas en el borojó. Moldovan et al., 2016, evaluaron la influencia de la temperatura y almacenamiento en los parámetros de degradación cinética de compuestos fenólicos de extractos de las drupas del Cornus mas, siendo estable el contenido fenólico hasta temperaturas de $22^{\circ} \mathrm{C}$, pudiendo almacenarse hasta dos meses a temperatura ambiente sin un significativo decrecimiento del contenido fenólico. Cabe señalar que no existen estudios sistemáticos disponibles que proporcionen información sobre la trasformación que sufren los compuestos fenólicos durante el almacenamiento y la forma en que afecta a su actividad biológica (Srivastava et al., 2007). Generalmente el contenido fenólico puede incrementarse o decrecer en frutas y vegetales dependiendo de las condiciones de almacenamiento (Dolatabadi et al., 2015). El presente estudio evalúo la estabilidad de las bebidas a temperaturas de 4 y $18{ }^{\circ} \mathrm{C}$, siendo la tendencia de mantenerse estables en el tiempo como se muestra en la Figura 2, similares a lo mencionado por Moldovan et al., 2016.

La estabilidad de la capacidad antioxidante CUPRAC y ABTS muestra la tendencia de permanecer estables en el tiempo en este tipo de matriz evaluada según se observa en las Figuras 3 y 4 ; en algunas bebidas de maíz morado estudiadas los valores de correlación son mayores que denotan una tendencia positiva en cuanto se refiere a la capacidad antioxidante CUPRAC y menores para ABTS en el tiempo evaluado. Sin embargo los valores de $k$ en todos los casos son muy bajos. Se desprende por lo anterior que para determinar la vida útil de este tipo de productos, deberían de tomarse en cuenta otros criterios como el contenido de antocianinas en las bebidas que expresa en mayor medida los procesos de degradación de la calidad en las bebidas. Resulta evidente que la larga vida útil a menudo que se aplica para de zumos comerciales resulta perjudicial para las antocianinas. Hellström et al., 2013 reportan el caso de que solo un 11-15\% del contenido original de antocianinas fue detectado en dos bebidas de zumos comerciales a su fecha de caducidad, después de un almacenamiento de 35-49 semanas a temperatura ambiente. La disminución del contenido de antocianinas en el almacenamiento y la poca variación en el contenido de fenoles y capacidad antioxidante a las temperaturas evaluadas y almacenadas durante 86 días de almacenamiento en ausencia de luz, sugieren la formación de otros tipos de compuestos fenólicos.
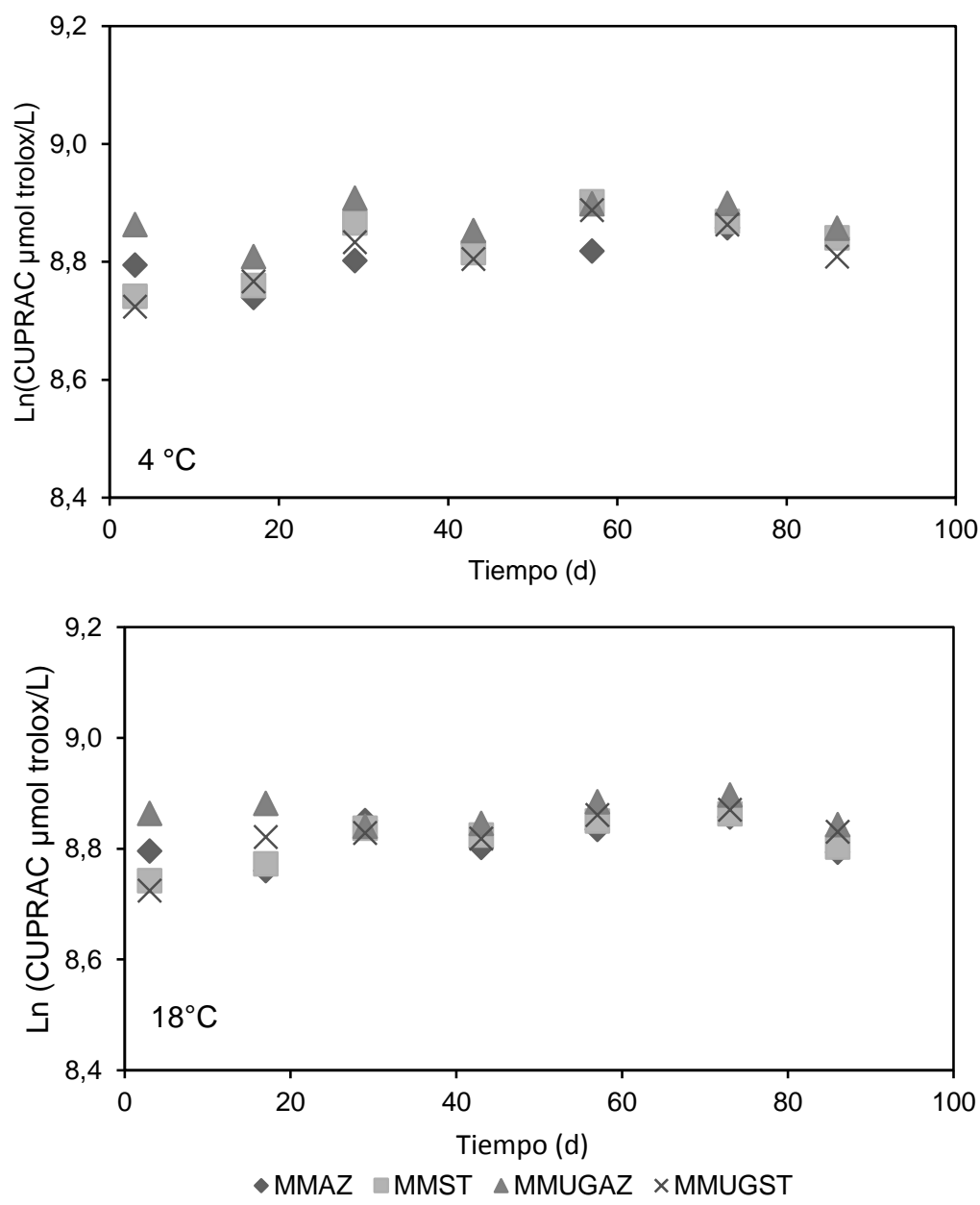

Fig. 3: Estabilidad de la CA CUPRAC en almacenamiento a 4 y $18^{\circ} \mathrm{C}$ 

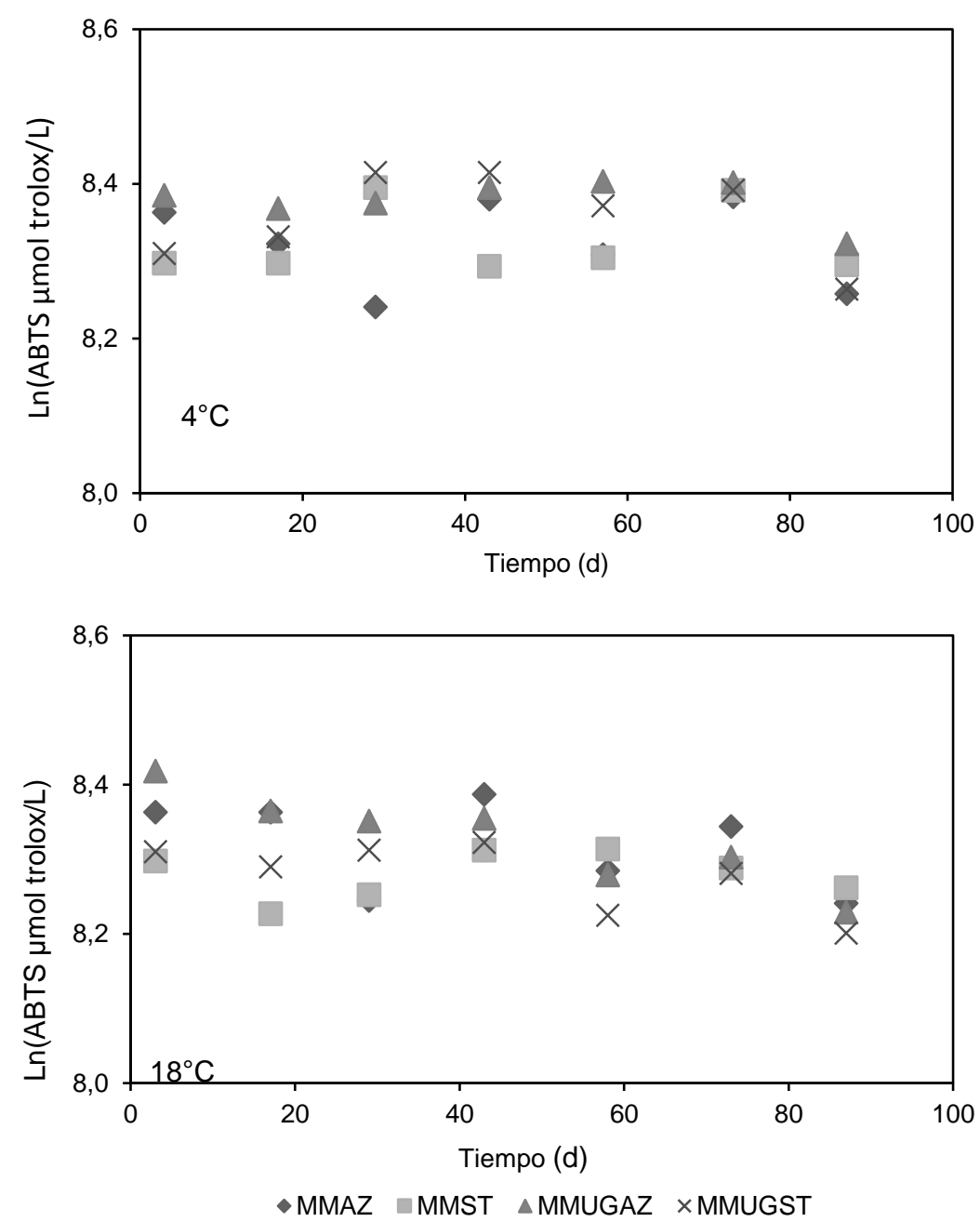

Fig. 4: Estabilidad de la CA ABTS en almacenamiento a 4 y $18^{\circ} \mathrm{C}$

Similares observaciones han sido señaladas por Pi-Jen et al., 2002, por el poco decrecimiento del total de compuestos fenólicos y de actividad antioxidante de extractos de la flor de Jamaica. Así mismo, las bajas temperaturas de almacenamiento contribuyen a la estabilidad de la capacidad antioxidante y de antocianinas frente a otras condiciones de mayor temperatura tanto en el procesamiento como en el almacenamiento en las cuales decrece (Sui et al., 2016). Las condiciones de calentamiento y de almacenamiento afectan significativamente el contenido de sustancias bioactivas, las pérdidas en fenoles totales, antocianinas y capacidad antioxidante son menores comparadas con los valores iniciales cuando se almacenan a bajas temperaturas en el caso de arándanos (Srivastava et al., 2007). En bebidas de sauco almacenadas a $6-8^{\circ} \mathrm{C}$ durante 90 días se alcanzó un porcentaje de retención de 86, 85, 96 y 95\% para las antocianinas, fenoles totales, capacidad antioxidante CUPRAC y ABTS respectivamente (Flores, 2017), lo cual muestra una mayor estabilidad en su capacidad antioxidante. Los estudios de estabilidad de las características antioxidantes de las bebidas por la presencia de sustancias bioactivas en su contenido son importantes tomarlas en consideración, toda vez que existe un gran interés por la calidad de las bebidas funcionales a expenderse a nivel industrial.

En cuanto se refiere a las características físico químicas en las bebidas elaboradas: MMAZ, MMST, MMUGAZ, MMUGST, estas se mantuvieron en el rango de 0.05- 0.06 para la acidez total expresada como \% de ácido cítrico, pH entre $3.9-4$ y ${ }^{\circ} \mathrm{Bx}$ entre 8 - 8.4 en las bebidas que contenían azúcar.

Los resultados de evaluación sensorial al aplicar la escala hedónica durante el periodo evaluado siguieron el siguiente orden de preferencia, los valores entre paréntesis corresponden a valores promedio de los puntajes otorgados por los jueces, hubo diferencia significativa entre la bebida de MMUGAZ y MMST. De acuerdo a la prueba de Tukey realizada con un nivel de significancia del $1 \%$.

a) Almacenamiento en refrigeración

MMUGAZ (6.86) >MMAZ (6.36)>MMUGST (6.29)>MMST (5.93)

b) Almacenamiento a temperatura ambiente 
MMUGAZ (6.96) >MMAZ (6.43)>MMUGST (6.11)>MMST (5.86)

Lo que indica que la bebida de mayor preferencia es la de MMUGAZ, siguen en orden las bebidas MMAZ, MMUGST y MMST.

En cuanto al sabor en relación a su vida útil, se tiene el siguiente orden:

c) Almacenamiento en refrigeración

MMUGAZ (5.79) >MMUGST (5.36)>MMAZ (5.21)>MMST (5.07)

d) Almacenamiento a temperatura ambiente

MMUGAZ (6.0) >MMAZ (5.43)>MMUGST (5.14)>MMST (4.89)

La bebida con mejor características de sabor es MMUGAZ, seguidas de MMAZ, MMUGST y MMST

El valor promedio en la escala hedónica está por encima de 4.5, lo que indica que todas las bebidas elaboradas son aceptables en cuanto al nivel de agrado (gustar y no gustar).

En cuanto al sabor del producto asociado a su vida útil todas las bebidas elaboradas, tienen un valor superior al límite de 3.5, lo que indica que los productos no han perdido sus características de calidad organoléptica en el periodo evaluado. La bebida de MMUGAZ presenta un sabor ligeramente tánico pero que sin embargo resalta favorablemente sus características organolépticas de sabor haciéndola diferente y con características particulares distintivas. En la bebida de MMUGST la presencia de uña de gato enmascara el gusto que comunica la stevia.

\section{CONCLUSIONES}

De los resultados mostrados, de su análisis y discusión se pueden obtener las siguientes conclusiones sobre la estabilidad de antocianinas, fenoles totales y capacidad antioxidante en las bebidas de maíz morado y uña de gato.

1) Las antocianinas sufrieron degradación durante el almacenamiento, siendo mayor la degradación a temperatura ambiente que en refrigeración siguiendo una cinética de primer orden. La bebida de MMUGAZ se degrada menos por lo cual es la que presenta mayor estabilidad

2) En general, el contenido de fenoles totales y la capacidad antioxidante en función del tiempo no mostraron variaciones importantes en las bebidas elaboradas.

3) Las bebidas elaboradas presentan características fisicoquímicas, sensoriales favorables, siendo interesante en este tipo de nuevas bebidas la conjugación de las propiedades benéficas de las mezclas de maíz morado y uña de gato.

\section{REFERENCIAS}

A.O.A.C. Oficial method of analysis. Association of Official Analiytical Chemistry. $16^{\text {th }}$ Ed., Ed. By Hoorwitz, N., P. Chialo, y H. Reynold, Washington, USA (1990)

Amaral, S., Mira, L., Nogueira, J., Pereira da Silva, A. y F. Florencio, Plant extracts with anti-inflammatory properties-A new approach for characterization of their bioactive compounds and establishment of structure-antioxidant activity relationships, doi: 10.1016/j.bmc.2009.01.045, Bioorganic \& Medicinal Chemistry 17, 1876-1883 (2009)

Anzaldua-Morales A., La evaluación sensorial de los alimentos en la teoría y la práctica. pp. 198, Editorial Acribia, S.A., Zaragosa, España (1994)

Arrazola, G., Herazo, I. y A. Alvis., Obtención y Evaluación de la Estabilidad de Antocianinas de Berenjena (Solanum melongena L.) en Bebidas, doi.org/10.4067/S0718-07642014000300007, Información Tecnológica, 25(3), 43-52 (2014)

Carmelo, G. y L. Sotelo, Efecto de las condiciones de almacenamiento sobre el color, contenido de polifenoles y capacidad antioxidante de una bebida de Borojoa patinoi Cuatrecasas, Boletín Latinoamericano y del Caribe de Plantas Medicinales y Aromáticas, https://goo.gl/LMuVNu, ISSN: 0717-7917, 11 (2), 196 - 205 (2012)

Cevallos -Casals, B. y L. Cisneros, Stoichiometric and Kinetic Studies of Phenolic Antioxidants from Andean Purple Corn and Red-Fleshed Sweetpotato, doi:10.1021/jf034109c, J. Agric. Food Chem. 2003, 51, 3313-3319 (2003)

Chang, R., Físico química para las ciencias químicas y biológicas, $3^{a}$ Ed., pp.1018, Editorial Mc Graw Hill, México D.F. (2008)

Damodaran, S., Kirk, L.P y O.R. Fennema, Química de los Alimentos. 3ª Ed., pp. 1154, Editorial Acribia, S.A Zaragosa España (2010)

Dolatabadi, M., Dehghan, G., Hosseini. S. y J. Esfahlan, Effect of five-year storage on total phenolic content and antioxidant capacity of almond (Amygdalus communis L.) hull and shell from different genotypes. https://goo.gl/LhaJ6A, Avicenna J. Phytomed, 5 (1), 26-33 (2015) 
Espinoza Atencia E., Evaluación Sensorial de los Alimentos, $1^{\text {a }}$ Ed., pp. 318, Universidad Nacional Jorge Basadre Grohmann, Tacna-Perú (2003)

Flores, E., Extracción de Antioxidantes de las Bayas del Sauco (Sambucus nigra L. subsp. peruviana) con Ultrasonido, Microondas, Enzimas y Maceración para la obtención de Zumos Funcionales, doi: 10.4067/S0718-07642017000100012, Información Tecnológica, 28 (1), 121-132 (2017)

Giusti, M. M., y R.E. Wrolstad, Acylated anthocyanins from edible sources and their applications in food systems, doi:10.1016/S1369-703X(02)00221-8, Biochemical Engineering Journal, 14, 217-225 (2003)

Guclu, K., Altun, M., Ozyurek, M., Karademir, S. y R. Apak., Antioxidant capacity of fresh, sun-and sulphited-dried Malatya apricot (Prunus armeniaca) assayed by CUPRAC, ABTS/TEAC and Folin methods, doi: 10.1111/j.13652621.2006.01347.x, International Journal of Food Science and technology, 41 (Supplement I) 76-85 (2006)

Hellström, J., Pirjo, M. y R. Karjalainen, Stability of anthocyanins in berry juices stored at different temperatures Journal of Food Composition and Analysis, doi.org/10.1016/j.jfca.2013.02.010, 31(1), 12-19 (2013)

Hurtado, N. y M. Pérez, Identificación, Estabilidad y Actividad Antioxidante de las Antocianinas Aisladas de la Cáscara del Fruto de Capulí (Prunus serotina spp capuli (Cav) Mc. Vaug Cav), doi: 10.4067/S0718 07642014000400015, Información Tecnológica, 25(4), 131-140 (2014)

Jing, P. y Giusti, M.M., Effects of extraction conditions on improving the Yield and Quality of an Anthocyanin-Rich Purple Corn (Zea mays L.) Color Extract, doi:10.1111/j.1750-3841.2007.004410x, Journal of Food Science, 72 (7), C363-8 (2007)

Laleh, G., Frydoonar, H., Heidary, R., Jameei, R. y S. Zare, The effect of light, temperature, pH and species on stability of anthocianyn pigments in four Berberis species, doi:10.3973/pjn.2006.90.92, Pakistan Journal and Nutrition, 5(1), 90-92 (2006)

Lemus-Mondaca, L., Vega-Gálvez A., Zura-Bravo L. y K.Ah-Hen, Stevia rebaudiana Bertoni, source of a high-potency natural sweetener: A comprehensive review on the biochemical, nutritional and functional aspects, doi:10.1016/j.foodchem.2011.11.140, Food Chemistry, 132(3), 1121-1132 (2012)

López, S., Márquez. y Pretell, C., Temperatura y tiempo de tratamiento térmico y el color de la pulpa de ají escabeche, https://goo.gl/qTTX2e, Revista Pueblo Continente, 20 (1) (2009)

Malien, A., Dangles, O., y M.J. Amiot., Color Stability of Commercial Anthocyanin-Based Extracts in Relation to the Phenolic Composition. Protective Effects by Intra- and Intermolecular Copigmentation, PMID:11170573, J. Agric. Food Chem., 49(1),170-176 (2001)

Martinez, J.J., Rojas, H., Borda, G., Hastamorir, A. y M. Riaño., Estabilidad de Antocianinas en Jugo y Concentrado de Agraz (Vaccinium meridionale Sw), https://goo.gl/bqSqoS, Rev. Fac. Nal. Agr. Medellín 64(1), 6015-6022 (2011)

Moldovan, B., Popa,A. y L. David, Effects of storage temperature on the total phenolic content of Cornelian Cherry (Cornus mas L.) fruits extracts, doi:105073/JABFQ.2016.089.026, Journal of Applied Botany and Food Quality 89, 208 - 211 (2016)

Navarro, M., Sánchez, F., Murillo, R., Martín, P. J., Zamora, W., Monagas, M. J. y B. Bartolomé, Phenolic assesment of Uncaria tomentosa L. (Cat's Claw): Leaves, stem, bark and wood extracts, doi:10.3390, Molecules, 20(12), 22703-22717 (2015)

Pi-Jen, T., McIntosh J. y P. Pearce, Anthocyanin and antioxidant capacity in Roselle (Hibiscus Sabdariffa L.) extract, Food Research International, 35(4), 351-356 (2002)

Pilarski R., Zielinski H., Ciesiołka D. y K. Gulewicz, Antioxidant activity of ethanolic and aqueous extracts of Uncaria tomentosa (Willd.) DC, doi:10.1016/j.jep.2005.08.046, Journal of Ethnopharmacology, 104 (1-2), 18-23 (2006)

Ramos, F., Muñoz, A. M., Alvarado, C., Alvarado, A. y J.A. Yánez, Purple corn (Zea mays L.) phenolic compounds profile and its assessment as an agent against oxidative stress in isolated mouse organs, DOI: 10.1089/jmf.2010.0342, Journal of medicinal food, 15(2), 206-215 (2012)

Rojas S.W., Stevia, edulcorante orgánico del siglo XXI. $1^{\circ}$ Ed., pp 391, Editado por la Universidad Nacional Agraria la Molina, Lima - Perú (2009)

Srivastava, A., Akoh,C., Yi, W., Fischer,J. y G. Krewer., Effect of Storage Conditions on the Biological Activity of Phenolic Compounds of Blueberry Extract Packed in Glass Bottles, doi:10.1021/jf062914w, J. Agric. Food Chem, 55 (7), pp. 2705$2713(2007)$

Sui, X., Bary, S. y W. Zhou, Changes in the color, chemical stability and antioxidant capacity of thermally treated anthocyanin aqueous solution over storage,doi:10.1016/j.foodchem.2015.07.021, Food Chemistry, 192 (1), $516-524$ (2016)

Valerio, L. y F. Gonzales, Toxicological Aspects of the South American Herbs Cat's Claw (Uncaria tomentosa) and Maca (Lepidium meyenii) A Critical Synopsis, PMID: 16042502, Toxicol. Rev., 24 (1), 11-35 (2005)

Wrolstad, R., Color and Pigment Analyses in Fruit Products. Oregon State University Agricultural Experiment Station Bulletin 624 (1976)

Yang, Z., Han, Y., Gu, Z., Fan, G. y Z. Chen, Thermal degradation kinetics of aqueous anthocyanins and visual color of purple corn(Zea mays L.) cob. Doi: 10.1016/j:ifset.2007.09.00, Innovative Food Science \& Emerging Technologies, 9(3), 341- 347 (2008) 